\title{
Global dynamics of a time-delayed echinococcosis transmission model
}

\author{
Junli Liu', Luju Liu², Xiaomei Feng ${ }^{3}$ and Jinqian Feng ${ }^{1}$
}

"Correspondence: lujuliu@126.com ${ }^{2}$ School of Mathematics and

Statistics, Henan University of Science and Technology, Luoyang, 471023, P.R. China

Full list of author information is available at the end of the article

\begin{abstract}
In this paper, we present a time-delayed echinococcosis transmission model to explore effective control and prevention strategies. We first give the basic reproduction number $R_{0}$. It is shown that if $R_{0}<1$, the disease-free equilibrium is globally asymptotically stable, and if $R_{0}>1$, the disease persists. We further show that the endemic equilibrium is globally asymptotically stable for a special case. Numerical simulations are performed to illustrate our analytic results. We give some sensitivity analysis of some parameters and give some useful comments on controlling the transmission of echinococcosis.
\end{abstract}

Keywords: echinococcosis transmission; uniform persistence; Lyapunov functional; global stability

\section{Introduction}

Echinococcosis, also called hydatid disease, hydatidosis, or echinococcal disease, is a parasitic disease of tapeworms of the Echinococcus type. The disease occurs in most areas of the world and currently affects about one million people. In some areas of South America, Africa, and Asia up to $10 \%$ of certain populations are affected [1]. In 2010, it caused about 1,200 deaths down from 2,000 in 1990 [2]. The economic cost of the disease is estimated to be around $3 \times 10^{9}$ USD a year. It can affect both humans and other animals such as pigs, cows, and horses [1]. The most common form found is cystic echinococcosis (also known as unilocular echinococcosis), which is caused by Echinococcus granulosus. The second most common form is alveolar echinococcosis, which is caused by Echinococcus multilocularis.

Like many other parasite infections, the course of Echinococcus infection is complex. The worm has a life cycle that requires definitive hosts and intermediate hosts. Definitive hosts are normally carnivores such as dogs, while intermediate hosts are usually herbivores such as sheep and cattle. Humans function as accidental hosts, because they are usually a dead-end for the parasitic infection cycle.

There are three development stages in the life cycle of Echinococcus, including egg, larva, and adult. An adult worm resides in the small intestine of a definitive host. Afterwards, gravid proglottids release eggs that are passed in the feces of the definitive host. The egg is then ingested by an intermediate host. The egg then hatches in the small intestine of the intermediate host and releases an oncosphere that penetrates the intestinal wall and moves through the circulatory system into different organs, in particular the liver

(c) 2015 Liu et al.: licensee Springer. This is an Open Access article distributed under the terms of the Creative Commons Attribution License (http://creativecommons.org/licenses/by/4.0), which permits unrestricted use, distribution, and reproduction in any medium, provided the original work is properly credited. 
and lungs. Once it has invaded these organs, the oncosphere develops into a cyst. The cyst then slowly enlarges, creating protoscolices and daughter cysts within the cyst. The definitive host then becomes infected after ingesting the cyst-containing organs of the infected intermediate host. After ingestion, the protoscolices attach to the intestine. They then develop into adult worms and the cycle starts all over again.

In China, there are 22 provinces, autonomous regions, and municipalities reported with cystic echinococcosis (CE) which was caused by Echinococcus granulosus and Echinococcus multilocularis [3, 4]. The main endemic areas are in the western and northwestern provinces and autonomous regions: Xinjiang, Gansu, Ningxia, Inner Mongolia, Qinghai, Tibet [5], and Sichuan [6, 7], where extensively developed livestock husbandry maintains stable transmission cycles of Echinococcus granulosus. The number of domestic animals being faced with the infection of echinococcosis is more than $10^{8}$, in which the amount of dogs is at least $5 \times 10^{6}[8]$.

Much has been done in terms of modeling and analysis of disease transmission of Echinococcus (see [9-13]). In [14], in order to explore effective control and prevention measures the authors proposed a deterministic model to study the transmission dynamics of echinococcosis in Xinjiang. The results showed that the dynamics of the model was completely determined by the basic reproductive number $R_{0}$. Du et al. [15] proposed an echinococcosis transmission model with saturation incidence, and they also established a threshold type result, which states that when $R_{0}<1$, the disease will die out; when $R_{0}>1$ and the recovery rate of dogs is very small, the disease will persist.

In this paper, we focus on the Echinococcus granulosus, which is the most common cause of human hydatid disease. The egg needs 5 to 6 months to develop into a larva in the intermediate hosts, and protoscoleces may develop into adult worms in about 1.5 to 2 months [16] in the definitive hosts. In view of realistic considerations, we take two time delays into account, to describe the time needed from egg to larva and from larva to adult, respectively. In fact, from the expression of $R_{0}$ in Section 2, we can see those delays reduce the values of $R_{0}$. Therefore, the neglect of the delays overestimated the infection risk.

The purpose of this paper is to study the global dynamics of a time-delayed Echinococcus transmission model. In Section 2, we present the model and prove its wellposedness, also we introduce the basic reproduction number $R_{0}$. In Section 3, we show the global stability of the disease-free equilibrium when $R_{0}<1$. In Section 4 , we show that the disease is uniformly persistent when $R_{0}>1$. In Section 5 , by constructing Lyapunov functionals, we show that the endemic equilibrium is globally asymptotically stable. In Section 6, we perform some sensitivity analysis of several model parameters and give some useful comments on controlling the transmission of echinococcosis.

\section{Model formulation}

We divide the definitive hosts population (mainly the dogs) into three subclasses: the susceptible population, the exposed population, and the infected population, denoted by $S_{1}(t), E_{1}(t)$, and $I_{1}(t)$, respectively, and $N_{1}(t)=S_{1}(t)+E_{1}(t)+I_{1}(t)$ is the total number of definitive hosts. The definitive hosts are infected by means of eating infected, cystcontaining organs.

We divide the intermediate hosts population into three subclasses: the susceptible population $\left(S_{2}(t)\right)$, the exposed population $\left(E_{2}(t)\right)$ and the infected population $\left(I_{2}(t)\right)$, and $N_{2}(t)=S_{2}(t)+E_{2}(t)+I_{2}(t)$ is the total number of intermediate hosts. The intermediate 
hosts are infected via the ingestion of eggs. Since eggs are released by the infected definitive hosts, we assume that the amount of eggs is proportional to the amount of infected definitive hosts. It follows from [14] that the parameters of the humans do not affect the dynamical behaviors of the echinococcosis model. Hence in the paper we only consider definitive hosts and intermediate hosts in our model.

An infectious individual can contact a finite number of individuals in one time unit in a large population. The standard incidence rate seems more reasonable than the bilinear incidence rate in [14]. Therefore, in our model, we discuss the dynamic behavior for an echinococcosis model with standard incidence rate. Then we take the following model:

$$
\begin{aligned}
& \frac{d S_{1}(t)}{d t}=A_{1}-\frac{\beta_{1} S_{1}(t) I_{2}(t)}{N_{1}(t)}-d_{1} S_{1}(t)+\sigma I_{1}(t), \\
& \frac{d E_{1}(t)}{d t}=\frac{\beta_{1} S_{1}(t) I_{2}(t)}{N_{1}(t)}-d_{1} E_{1}(t)-\beta_{1} e^{-d_{1} \tau_{1}} \frac{S_{1}\left(t-\tau_{1}\right) I_{2}\left(t-\tau_{1}\right)}{N_{1}\left(t-\tau_{1}\right)}, \\
& \frac{d I_{1}(t)}{d t}=\beta_{1} e^{-d_{1} \tau_{1}} \frac{S_{1}\left(t-\tau_{1}\right) I_{2}\left(t-\tau_{1}\right)}{N_{1}\left(t-\tau_{1}\right)}-\left(d_{1}+\sigma\right) I_{1}(t), \\
& \frac{d S_{2}(t)}{d t}=A_{2}-\frac{\beta_{2} S_{2}(t) I_{1}(t)}{N_{2}(t)}-d_{2} S_{2}(t), \\
& \frac{d E_{2}(t)}{d t}=\frac{\beta_{2} S_{2}(t) I_{1}(t)}{N_{2}(t)}-d_{2} E_{2}(t)-\beta_{2} e^{-d_{2} \tau_{2}} \frac{S_{2}\left(t-\tau_{2}\right) I_{1}\left(t-\tau_{2}\right)}{N_{2}\left(t-\tau_{2}\right)}, \\
& \frac{d I_{2}(t)}{d t}=\beta_{2} e^{-d_{2} \tau_{2}} \frac{S_{2}\left(t-\tau_{2}\right) I_{1}\left(t-\tau_{2}\right)}{N_{2}\left(t-\tau_{2}\right)}-\left(d_{2}+\varepsilon_{2}\right) I_{2}(t) .
\end{aligned}
$$

All parameters are assumed nonnegative. For the definitive hosts population, $A_{1}$ describes the annual recruitment rate; $d_{1}$ is the natural death rate; $\sigma$ denotes the recovery rate of transition from infected to susceptible definitive hosts, including the natural recovery rate and recovery due to anthelmintic treatment; $\frac{\beta_{1} S_{1}(t) I_{2}(t)}{N_{1}(t)}$ describes the transmission of echinococcosis between susceptible definitive hosts and infectious intermediate hosts after the ingestion of cyst-containing organs of the infected intermediate hosts. For the intermediate hosts, $A_{2}$ is the annual recruitment rate; $d_{2}$ is the death rate; $\frac{\beta_{2} S_{2}(t) I_{1}(t)}{N_{2}(t)}$ describes the transmission of echinococcosis to intermediate hosts by the ingestion of Echinococcus eggs in the environment, $\varepsilon_{2}$ is the death-induced death rate. $\tau_{1}$ is the time needed for eggs to develop into larvae in the intermediate hosts, and $\tau_{2}$ is the time needed for protoscoleces to develop into adult worms in the definitive hosts.

It is easy to see that the equations for $E_{1}(t)$ and $E_{2}(t)$ can be rewritten as two integral equations:

$$
\begin{aligned}
& E_{1}(t)=\int_{t-\tau_{1}}^{t} e^{-d_{1}(t-\theta)} \frac{\beta_{1} S_{1}(\theta) I_{2}(\theta)}{N_{1}(\theta)} d \theta, \\
& E_{2}(t)=\int_{t-\tau_{2}}^{t} e^{-d_{2}(t-\theta)} \frac{\beta_{2} S_{2}(\theta) I_{1}(\theta)}{N_{2}(\theta)} d \theta .
\end{aligned}
$$

Let $\tau=\max \left\{\tau_{1}, \tau_{2}\right\}$, in view of (2) and (3), the initial conditions for system (1) take the form of

$$
\begin{array}{lll}
S_{1}(\theta)=\phi_{1}(\theta) \geq 0, & E_{1}(\theta)=\phi_{2}(\theta) \geq 0, & I_{1}(\theta)=\phi_{3}(\theta) \geq 0, \\
S_{2}(\theta)=\phi_{4}(\theta) \geq 0, & E_{2}(\theta)=\phi_{5}(\theta) \geq 0, & I_{2}(\theta)=\phi_{6}(\theta) \geq 0, \quad \theta \in[-\tau, 0],
\end{array}
$$


and

$$
\begin{aligned}
& \sum_{i=1}^{3} \phi_{i}(\theta)>0, \quad \sum_{i=4}^{6} \phi_{i}(\theta)>0, \quad \forall \theta \in[-\tau, 0] \\
& \phi_{2}(0)=\int_{-\tau_{1}}^{0} e^{d_{1} \theta} \frac{\beta_{1} \phi_{1}(\theta) \phi_{6}(\theta)}{\sum_{i=1}^{3} \phi_{i}(\theta)} d \theta \\
& \phi_{5}(0)=\int_{-\tau_{2}}^{0} e^{d_{2} \theta} \frac{\beta_{2} \phi_{4}(\theta) \phi_{3}(\theta)}{\sum_{i=4}^{6} \phi_{i}(\theta)} d \theta
\end{aligned}
$$

where $\Phi=\left(\phi_{1}, \phi_{2}, \phi_{3}, \phi_{4}, \phi_{5}, \phi_{6}\right) \in C^{+}\left([-\tau, 0], \mathbb{R}_{+}^{6}\right)$, the space of continuous functions mapping $[-\tau, 0]$ into $\mathbb{R}_{+}^{6}$.

By a similar proof to Theorem 1 of [14], we can show the following.

Lemma 2.1 The solutions of system (1) with initial conditions (4) and (5) satisfy $S_{1}(t)>0$, $E_{1}(t) \geq 0, I_{1}(t) \geq 0, S_{2}(t)>0, E_{2}(t) \geq 0, I_{2}(t) \geq 0$ for all $t>0$.

For any $\varepsilon>0$, we define $\Omega_{\varepsilon}$ as

$$
\Omega_{\varepsilon}=\left\{\left(S_{1}, E_{1}, I_{1}, S_{2}, E_{2}, I_{2}\right) \in \mathbb{R}_{+}^{6}: S_{1}+E_{1}+I_{1} \leq \frac{A_{1}}{d_{1}}+\varepsilon, S_{2}+E_{2}+I_{2} \leq \frac{A_{2}}{d_{2}}+\varepsilon\right\} .
$$

Lemma 2.2 All solutions of system (1) with initial conditions (4) and (5) ultimately turn into region $\Omega_{\varepsilon}$ as $t \rightarrow \infty$.

Proof From system (1), we have

$$
\begin{aligned}
& \frac{d N_{1}(t)}{d t}=A_{1}-d_{1} N_{1}(t) \\
& \frac{d N_{2}(t)}{d t}=A_{2}-d_{2} N_{2}(t)-\varepsilon_{2} I_{2}(t) \leq A_{2}-d_{2} N_{2}(t) .
\end{aligned}
$$

For the system

$$
\frac{d x(t)}{d t}=A_{i}-d_{i} x(t), \quad i=1,2,
$$

the equilibrium $x^{*}=\frac{A_{i}}{d_{i}}$ is globally asymptotically stable. By the comparison principle, it follows that

$$
\limsup _{t \rightarrow \infty}\left(N_{1}(t), N_{2}(t)\right) \leq\left(\frac{A_{1}}{d_{1}}, \frac{A_{2}}{d_{2}}\right) .
$$

Hence, for any $\varepsilon>0$, there is a $t_{1}>0$ such that

$$
\begin{aligned}
& S_{1}(t)+E_{1}(t)+I_{1}(t)=N_{1}(t) \leq \frac{A_{1}}{d_{1}}+\varepsilon, \\
& S_{2}(t)+E_{2}(t)+I_{2}(t)=N_{2}(t) \leq \frac{A_{2}}{d_{2}}+\varepsilon \quad \text { for all } t \geq t_{1} .
\end{aligned}
$$

This completes the proof. 
Remark 2.1 Lemma 2.2 tells us that all feasible solutions of model (1) enter or remain in the region $\Omega_{\varepsilon}$ as $t$ becomes large enough. Hence, the dynamics of model (1) can be considered only in $\Omega_{\varepsilon}$.

It is easy to check that $E_{0}=\left(\frac{A_{1}}{d_{1}}, 0,0, \frac{A_{2}}{d_{2}}, 0,0\right)$ is the disease-free equilibrium of (1), and it exists for all nonnegative values of the parameters. According to the idea in [17], we obtain the basic reproduction number

$$
R_{0}=\sqrt{\frac{\beta_{1} \beta_{2} e^{-d_{1} \tau_{1}} e^{-d_{2} \tau_{2}}}{\left(d_{1}+\sigma\right)\left(d_{2}+\varepsilon_{2}\right)}} .
$$

Remark 2.2 Near the disease-free equilibrium $E_{0}$, each infected intermediate host produces $\frac{\beta_{1} e^{-d_{1} \tau_{1}}}{d_{2}+\varepsilon_{2}}$ new infected definitive hosts over its expected infectious period, and each definitive host produces $\frac{\beta_{2} e^{-d_{2} \tau_{2}}}{d_{1}+\sigma}$ new infected intermediate hosts over its expected infectious period. The square root arises from the two 'generations' required for an infected definitive host or intermediate host to 'reproduce' itself.

\section{Global stability of $E_{0}$}

For the disease-free equilibrium $E_{0}$, we will show that the disease dies out if $R_{0}<1$.

Theorem 3.1 The disease-free equilibrium $E_{0}=\left(\frac{A_{1}}{d_{1}}, 0,0, \frac{A_{2}}{d_{2}}, 0,0\right)$ is unstable if $R_{0}>1$, and it is globally asymptotically stable if $R_{0}<1$.

Proof The characteristic equation of system (1) at $E_{0}$ is

$$
\left(\lambda+d_{1}\right)^{2}\left(\lambda+d_{2}\right)^{2} f(\lambda)=0
$$

where

$$
f(\lambda)=\lambda^{2}+\left(d_{1}+\sigma+d_{2}+\varepsilon_{2}\right) \lambda+\left(d_{1}+\sigma\right)\left(d_{2}+\varepsilon_{2}\right)-\beta_{1} \beta_{2} e^{-d_{1} \tau_{1}} e^{-d_{2} \tau_{2}} e^{-\lambda\left(\tau_{1}+\tau_{2}\right)} .
$$

Clearly, (6) has four negative roots $\lambda_{1}=\lambda_{2}=-d_{1}, \lambda_{3}=\lambda_{4}=-d_{2}$. Therefore, the stability of $E_{0}$ is determined by the distribution of roots of $f(\lambda)=0$.

Note that if $R_{0}=\sqrt{\frac{\beta_{1} \beta_{2} e^{-d_{1} \tau_{1}} e^{-d_{2} \tau_{2}}}{\left(d_{1}+\sigma\right)\left(d_{2}+\varepsilon_{2}\right)}}>1$, then $f(0)=\left(d_{1}+\sigma\right)\left(d_{2}+\varepsilon_{2}\right)-\beta_{1} \beta_{2} e^{-d_{1} \tau_{1}} e^{-d_{2} \tau_{2}}<0$, and $f(+\infty)=\infty$. Hence, $f(\lambda)=0$ has at least one positive root and $E_{0}$ is unstable.

If $R_{0}<1$, we define $m_{1}=d_{1}+\sigma+d_{2}+\varepsilon_{2}, m_{2}=\left(d_{1}+\sigma\right)\left(d_{2}+\varepsilon_{2}\right)$. Let $\lambda=u+i v$ with $u, v \in \mathbb{R}$ be a root of $f(\lambda)=0$. Then we have

$$
\begin{aligned}
& u^{2}-v^{2}+m_{1} u+m_{2}=R_{0}^{2} m_{2} e^{-u\left(\tau_{1}+\tau_{2}\right)} \cos \left(\left(\tau_{1}+\tau_{2}\right) v\right), \\
& 2 u v+m_{1} v=-R_{0}^{2} m_{2} e^{-u\left(\tau_{1}+\tau_{2}\right)} \sin \left(\left(\tau_{1}+\tau_{2}\right) v\right),
\end{aligned}
$$

thus,

$$
\begin{aligned}
& \left(u^{2}+v^{2}\right)^{2}+2 u^{2}\left(m_{1} u+m_{2}\right)+\left(m_{1} u+m_{2}\right)^{2}+2 m_{1} u v^{2}+\left[\left(d_{1}+\sigma\right)^{2}+\left(d_{2}+\varepsilon_{2}\right)^{2}\right] v^{2} \\
& \quad=R_{0}^{4} m_{2}^{2} e^{-2 u\left(\tau_{1}+\tau_{2}\right)} .
\end{aligned}
$$


If $u \geq 0$, then each term in the left hand side of (7) is nonnegative, furthermore, $\left(m_{1} u+\right.$ $\left.m_{2}\right)^{2} \geq m_{2}^{2}$, while for the right hand side of (7), $R_{0}^{4} m_{2}^{2} e^{-2 u\left(\tau_{1}+\tau_{2}\right)} \leq R_{0}^{4} m_{2}^{2}<m_{2}^{2}$, a contradiction. This shows that all roots of $f(\lambda)=0$ must have negative real parts. Therefore, $E_{0}$ is locally asymptotically stable.

To complete the proof of Theorem 3.1, we only need to show that $E_{0}$ is globally attractive under the condition $R_{0}<1$. From system (1), we obtain

$$
\begin{aligned}
& \frac{d I_{1}(t)}{d t} \leq \beta_{1} e^{-d_{1} \tau_{1}} I_{2}\left(t-\tau_{1}\right)-\left(d_{1}+\sigma\right) I_{1}(t), \\
& \frac{d I_{2}(t)}{d t} \leq \beta_{2} e^{-d_{2} \tau_{2}} I_{1}\left(t-\tau_{2}\right)-\left(d_{2}+\varepsilon_{2}\right) I_{2}(t) .
\end{aligned}
$$

Consider the following linear system:

$$
\begin{aligned}
& \frac{d x_{1}(t)}{d t}=\beta_{1} e^{-d_{1} \tau_{1}} x_{2}\left(t-\tau_{1}\right)-\left(d_{1}+\sigma\right) x_{1}(t), \\
& \frac{d x_{2}(t)}{d t}=\beta_{2} e^{-d_{2} \tau_{2}} x_{1}\left(t-\tau_{2}\right)-\left(d_{2}+\varepsilon_{2}\right) x_{2}(t),
\end{aligned}
$$

this is a cooperative and irreducible system of delay differential equations with a unique equilibrium $(0,0)$; the characteristic equation at $(0,0)$ is

$$
f(\lambda)=0 .
$$

Since all roots of $f(\lambda)=0$ have negative real parts, $E_{0}$ is locally asymptotically stable. Therefore,

$$
\lim _{t \rightarrow \infty}\left(x_{1}(t), x_{2}(t)\right)=(0,0)
$$

An application of the standard comparison argument yields

$$
\lim _{t \rightarrow \infty}\left(I_{1}(t), I_{2}(t)\right)=(0,0) .
$$

Hence, we have the following limiting system:

$$
\begin{aligned}
& \frac{d S_{1}(t)}{d t}=A_{1}-d_{1} S_{1}(t), \\
& \frac{d E_{1}(t)}{d t}=-d_{1} E_{1}(t), \\
& \frac{d S_{2}(t)}{d t}=A_{2}-d_{2} S_{2}(t), \\
& \frac{d E_{2}(t)}{d t}=-d_{2} E_{2}(t),
\end{aligned}
$$

which implies that

$$
\lim _{t \rightarrow \infty}\left(S_{1}(t), E_{1}(t), S_{2}(t), E_{2}(t)\right)=\left(\frac{A_{1}}{d_{1}}, 0, \frac{A_{2}}{d_{2}}, 0\right) .
$$


Thus, according to the theory of asymptotically autonomous semiflows [18], we find that the disease-free equilibrium $E_{0}$ is globally asymptotically stable if $R_{0}<1$. This completes the proof.

\section{Uniform persistence}

Using the method in [17], we now consider the issue of disease persistence.

Theorem 4.1 If $R_{0}>1$, then there exists an $\eta>0$ such that every solution $\left(S_{1}(t), E_{1}(t), I_{1}(t)\right.$, $\left.S_{2}(t), E_{2}(t), I_{2}(t)\right)$ of system (1) with initial conditions (4) and (5), $\phi_{3}(0) \neq 0, \phi_{6}(0) \neq 0$ satisfies

$$
\liminf _{t \rightarrow \infty}\left(I_{1}(t), I_{2}(t)\right) \geq(\eta, \eta)
$$

Proof Let

$$
\begin{aligned}
& X=\left\{\phi=\left(\phi_{1}, \phi_{2}, \phi_{3}, \phi_{4}, \phi_{5}, \phi_{6}\right) \in C^{+}\left([-\tau, 0], \mathbb{R}_{+}^{6}\right) \mid \phi \text { satisfies condition (5) }\right\}, \\
& X_{0}=\left\{\phi \in X \mid \phi_{3}(0) \neq 0, \phi_{6}(0) \neq 0\right\} .
\end{aligned}
$$

It follows that

$$
\partial X_{0}=X \backslash X_{0}=\left\{\phi \in X \mid \phi_{3}(0)=0 \text { or } \phi_{6}(0)=0\right\} .
$$

We also define

$$
M_{\partial}=\left\{\phi \in X \mid \Phi(t) \phi \in \partial X_{0}, \forall t \geq 0\right\} .
$$

Let $u_{t}$ be the solution of (1), let $\Phi(t): X \rightarrow X$ be the solution semiflow associated with (1); that is, $\Phi(t) \phi=u_{t}(\phi), \phi \in X, t \geq 0$. By Lemmas 2.1 and 2.2, the solutions of (1) are ultimately bounded, thus the semiflow $\Phi(t)$ is point dissipative on $X$, and $\Phi(t): X \rightarrow X$ is compact for all $t>\tau$. By [19], it then follows that $\Phi(t)$ admits a global attractor, which attracts every bounded set in $X$.

Claim 1: There is a $\delta>0$, such that, for any $\phi \in X_{0}$,

$$
\limsup _{t \rightarrow \infty}\left\|\Phi(t) \phi-E_{0}\right\| \geq \delta
$$

First we consider the following system:

$$
\begin{aligned}
& \frac{d y_{1}(t)}{d t}=\beta_{1} e^{-d_{1} \tau_{1}}(1-\varepsilon) y_{2}\left(t-\tau_{1}\right)-\left(d_{1}+\sigma\right) y_{1}(t), \\
& \frac{d y_{2}(t)}{d t}=\beta_{2} e^{-d_{2} \tau_{2}}(1-\varepsilon) y_{1}\left(t-\tau_{2}\right)-\left(d_{2}+\varepsilon_{2}\right) y_{2}(t) .
\end{aligned}
$$

For sufficiently small $\varepsilon>0$, let $\lambda_{1}(\varepsilon)$ be the principal eigenvalue of system (10). Since $R_{0}>1$, by Corollary 5.5.2 in [20] we have $\lambda_{1}(0)>0$. We then can restrict $\varepsilon$ to be small enough such that $\lambda_{1}(\varepsilon)>0$. For this $\varepsilon$, there is a $\delta>0$ such that

$$
\frac{x_{1}}{x_{1}+x_{2}+x_{3}}>1-\varepsilon>0
$$


and

$$
\frac{x_{4}}{x_{4}+x_{5}+x_{6}}>1-\varepsilon>0, \quad \forall\left|\left(x_{1}, x_{2}, x_{3}, x_{4}, x_{5}, x_{5}\right)-E_{0}\right|<\delta
$$

Suppose (9) does not hold, then there is a $\phi \in X_{0}$, such that

$$
\limsup _{t \rightarrow \infty}\left\|\Phi(t) \phi-E_{0}\right\|<\delta
$$

Then there exists a $t_{2}>t_{1}$, such that for all $t \geq t_{2}$, we have

$$
\left\|\Phi(t) \phi-E_{0}\right\|<\delta
$$

For any $\varepsilon>0$, we can choose $t_{3}>t_{2}$, such that for all $t \geq t_{3}$,

$$
\frac{S_{1}(t)}{N_{1}(t)}>1-\varepsilon, \quad \frac{S_{2}(t)}{N_{2}(t)}>1-\varepsilon .
$$

From system (1), when $t \geq t_{3}$, we have

$$
\begin{aligned}
& \frac{d I_{1}(t)}{d t} \geq \beta_{1} e^{-d_{1} \tau_{1}}(1-\varepsilon) I_{2}\left(t-\tau_{1}\right)-\left(d_{1}+\sigma\right) I_{1}(t), \\
& \frac{d I_{2}(t)}{d t} \geq \beta_{2} e^{-d_{2} \tau_{2}}(1-\varepsilon) I_{1}\left(t-\tau_{2}\right)-\left(d_{2}+\varepsilon_{2}\right) I_{2}(t) .
\end{aligned}
$$

Let $\left(v_{1}, v_{2}\right)^{T}$ be the positive right eigenvector associated with $\lambda_{1}(\varepsilon)$ for system (10), we can choose $r>0$ small enough such that

$$
r v_{1} e^{\lambda_{1}(\varepsilon) t} \leq I_{1}(t), \quad r v_{2} e^{\lambda_{1}(\varepsilon) t} \leq I_{2}(t), \quad \forall t \in\left[t_{3}, t_{3}+\tau\right]
$$

It is easy to see that $r e^{\lambda_{1}(\varepsilon) t}\left(v_{1}, v_{2}\right)^{T}$ satisfies (10) for $t \geq t_{3}$. Then by the comparison principle, we get

$$
\left(I_{1}(t), I_{2}(t)\right) \geq r e^{\lambda_{1}(\varepsilon) t}\left(v_{1}, v_{2}\right) \quad \text { for all } t \geq t_{3} .
$$

Since $\lambda_{1}(\varepsilon)>0$, we have $\lim _{t \rightarrow \infty}\left(I_{1}(t), I_{2}(t)\right)=(\infty, \infty)$, a contradiction to (11). Thus (9) holds.

Denote the $\omega$-limit set of the solution of system (1) starting in $\phi \in X$ by $\omega(\phi)$.

Claim 2: $\bigcup_{\phi \in M_{\partial}} \omega(\phi)=E_{0}$.

For any $\phi \in M_{\partial}$, we have $I_{1}(t, \phi) \equiv 0$ or $I_{2}(t, \phi) \equiv 0$. If $I_{1}(t, \phi) \equiv 0$, then from system (1), we get $\lim _{t \rightarrow \infty} S_{2}(t, \phi)=\frac{A_{2}}{d_{2}}, \lim _{t \rightarrow \infty} E_{2}(t, \phi)=0, \lim _{t \rightarrow \infty} I_{2}(t, \phi)=0$. By the theory of asymptotically autonomous semiflows [18], it follows that $\lim _{t \rightarrow \infty} S_{1}(t, \phi)=\frac{A_{1}}{d_{1}}$, $\lim _{t \rightarrow \infty} E_{1}(t, \phi)=0$. If $I_{2}(t, \phi) \equiv 0$, again from system $(1)$, we get $\lim _{t \rightarrow \infty} E_{1}(t, \phi)=0$, $\lim _{t \rightarrow \infty} I_{1}(t, \phi)=0$; furthermore, we obtain $\lim _{t \rightarrow \infty} S_{1}(t, \phi)=\frac{A_{1}}{d_{1}}, \lim _{t \rightarrow \infty} S_{2}(t, \phi)=\frac{A_{2}}{d_{2}}$, $\lim _{t \rightarrow \infty} E_{2}(t, \phi)=0$. Therefore, we have $\bigcup_{\phi \in M_{\partial}} \omega(\phi)=E_{0}$. 
Define $p: X \rightarrow \mathbb{R}_{+}$by

$$
p(\Phi)=\min \left\{\phi_{3}(0), \phi_{6}(0)\right\}, \quad \forall \Phi \in X .
$$

It is easy to see that $p^{-1}(0, \infty) \subset X_{0}$. By (1),

$$
I_{1}(t)=e^{-\left(d_{1}+\sigma\right) t}\left[I_{1}(0)+\int_{0}^{t} \beta_{1} e^{-d_{1} \tau_{1}} e^{\left(d_{1}+\sigma\right) \rho} \frac{S_{1}\left(\rho-\tau_{1}\right) I_{2}\left(\rho-\tau_{1}\right)}{N_{1}\left(\rho-\tau_{1}\right)} d \rho\right]
$$

and

$$
I_{2}(t)=e^{-\left(d_{2}+\varepsilon_{2}\right) t}\left[I_{2}(0)+\int_{0}^{t} \beta_{2} e^{-d_{2} \tau_{2}} e^{\left(d_{2}+\varepsilon_{2}\right) \rho} \frac{S_{2}\left(\rho-\tau_{2}\right) I_{1}\left(\rho-\tau_{2}\right)}{N_{2}\left(\rho-\tau_{2}\right)} d \rho\right] .
$$

Then $I_{i}(t)>0$ for all $t>0, i=1,2, \phi_{3}(0) \neq 0, \phi_{6}(0) \neq 0$. It follows that $p$ has the property that if either $p(\phi)=0$ and $\phi \in \partial X_{0}$, or $p(\phi)>0$, then $p(\Phi(t)(\phi))>0$ for all $t>0$. Hence, $p$ is a generalized distance function for the semiflow $\Phi(t): X \rightarrow X$ (see [21]). By Claim 2, we see that any forward orbit of $\Phi(t)$ in $M_{\partial}$ converges to $E_{0}$. By Claim 1, we see that $E_{0}$ is an isolated invariant set in $X$, and that $W^{s}\left(E_{0}\right) \cap X_{0}=\emptyset$, where $W^{s}\left(E_{0}\right)$ is the stable manifold of $E_{0}$. By [21], it then follows that there exists an $\eta>0$ such that $\liminf _{t \rightarrow \infty} p(\Phi(t) \phi) \geq \eta$ for any $\phi \in X_{0}$. This implies that $\liminf _{t \rightarrow \infty} I_{i}(t) \geq \eta, i=1,2$. This completes the proof.

\section{Global stability of endemic equilibrium}

In this section, we will study the global stability of endemic equilibrium of system (1). For simplicity, we assume that $\varepsilon_{2}=0$, we find that when $R_{0}>1$, system (1) has one endemic equilibrium; when $R_{0} \leq 1$, there is no endemic equilibrium, system (1) has only the diseasefree equilibrium $E_{0}$.

Theorem 5.1 Assume that $\varepsilon_{2}=0$. If $R_{0}>1$, system (1) has a unique endemic (positive) equilibrium $E^{*}=\left(S_{1}^{*}, E_{1}^{*}, I_{1}^{*}, S_{2}^{*}, E_{2}^{*}, I_{2}^{*}\right)$. More specifically,

(i) If $\sigma>0, E^{*}=\left(S_{1}^{*}, E_{1}^{*}, I_{1}^{*}, S_{2}^{*}, E_{2}^{*}, I_{2}^{*}\right)$ is given by

$$
\begin{aligned}
S_{1}^{*}= & \frac{A_{1}\left(A_{1}+\sigma I_{1}^{*}\right)}{d_{1}\left(A_{1}+\beta_{1} I_{2}^{*}\right)}, \\
S_{2}^{*}= & \frac{A_{2}^{2}}{d_{2}\left(A_{2}+\beta_{2} I_{1}^{*}\right)}, \\
E_{1}^{*}= & \frac{\beta_{1}\left(1-e^{-d_{1} \tau_{1}}\right) S_{1}^{*} I_{2}^{*}}{A_{1}}, \\
E_{2}^{*}= & \frac{\beta_{2}\left(1-e^{-d_{2} \tau_{2}}\right) S_{2}^{*} I_{1}^{*}}{A_{2}}, \\
I_{1}^{*}= & \frac{-a_{1}+\sqrt{a_{1}^{2}-4 a_{0} a_{2}}}{2 a_{0}}, \\
I_{2}^{*}= & \frac{\sigma\left[R_{0}^{2} A_{2}\left(d_{1}+\sigma-\sigma e^{-d_{1} \tau_{1}}\right)+\beta_{2} A_{1} e^{-d_{1} \tau_{1}}\right] I_{1}^{*}}{\beta_{1} \beta_{2} A_{1} e^{-d_{1} \tau_{1}}+\beta_{1} A_{2}\left(d_{1}+\sigma-\sigma e^{-d_{1} \tau_{1}}\right)} \\
& +\frac{\left(d_{1}+\sigma-\sigma e^{-d_{1} \tau_{1}}\right)\left(R_{0}^{2}-1\right) A_{1} A_{2}}{\beta_{1} \beta_{2} A_{1} e^{-d_{1} \tau_{1}}+\beta_{1} A_{2}\left(d_{1}+\sigma-\sigma e^{-d_{1} \tau_{1}}\right)},
\end{aligned}
$$


where

$$
\begin{aligned}
& a_{0}=\beta_{1} A_{2} \sigma R_{0}^{2}\left(d_{1}+\sigma-\sigma e^{-d_{1} \tau_{1}}\right)+\beta_{1} \beta_{2} A_{1} \sigma e^{-d_{1} \tau_{1}}, \\
& a_{1}=\beta_{1} A_{1} A_{2}\left[\sigma e^{-d_{1} \tau_{1}}\left(1-R_{0}^{2}\right)+R_{0}^{2}\left(d_{1}+\sigma-\sigma e^{-d_{1} \tau_{1}}\right)\right]+\beta_{1} \beta_{2} A_{1}^{2} e^{-d_{1} \tau_{1}}, \\
& a_{2}=A_{1}^{2} A_{2} \beta_{1} e^{-d_{1} \tau_{1}}\left(1-R_{0}^{2}\right) .
\end{aligned}
$$

(ii) If $\sigma=0, E^{*}=\left(S_{1}^{*}, E_{1}^{*}, I_{1}^{*}, S_{2}^{*}, E_{2}^{*}, I_{2}^{*}\right)$ is given by

$$
\begin{aligned}
& S_{1}^{*}=\frac{A_{1} d_{2}\left(A_{1} \beta_{2}+A_{2} d_{1} e^{d_{1} \tau_{1}}\right)}{d_{1} \beta_{2}\left(A_{1} d_{2}+A_{2} \beta_{1} e^{-d_{2} \tau_{2}}\right)}, \\
& S_{2}^{*}=\frac{A_{2} d_{1}\left(A_{2} \beta_{1}+A_{1} d_{2} e^{d_{2} \tau_{2}}\right)}{d_{2} \beta_{1}\left(A_{2} d_{1}+A_{1} \beta_{2} e^{-d_{1} \tau_{1}}\right)}, \\
& E_{1}^{*}=\frac{A_{1} A_{2} d_{2}\left(e^{d_{1} \tau_{1}}-1\right)\left(R_{0}^{2}-1\right)}{\beta_{2}\left(A_{1} d_{2}+A_{2} \beta_{1} e^{-d_{2} \tau_{2}}\right)}, \\
& E_{2}^{*}=\frac{A_{1} A_{2} d_{1}\left(e^{d_{2} \tau_{2}}-1\right)\left(R_{0}^{2}-1\right)}{\beta_{1}\left(A_{2} d_{1}+A_{1} \beta_{2} e^{-d_{1} \tau_{1}}\right)}, \\
& I_{1}^{*}=\frac{A_{1} A_{2} d_{2}\left(R_{0}^{2}-1\right)}{\beta_{1} \beta_{2} A_{2} e^{-d_{2} \tau_{2}}+\beta_{2} A_{1} d_{2}}, \\
& I_{2}^{*}=\frac{A_{1} A_{2} d_{1}\left(R_{0}^{2}-1\right)}{\beta_{1} \beta_{2} A_{1} e^{-d_{1} \tau_{1}}+\beta_{1} A_{2} d_{1}} .
\end{aligned}
$$

For the special case $\varepsilon_{2}=\sigma=0$, that is, the disease-induced death rate of infected livestock population is zero $\left(\varepsilon_{2}=0\right)$, we also assume that the infected dogs will not recover $(\sigma=0)$. In this case, as regards the stability of the endemic equilibrium, we have the following theorems.

Theorem 5.2 Assume that $\varepsilon_{2}=\sigma=0$. If $R_{0}>1$, the endemic equilibrium $E^{*}=\left(S_{1}^{*}, E_{1}^{*}, I_{1}^{*}, S_{2}^{*}\right.$, $\left.E_{2}^{*}, I_{2}^{*}\right)$ of system (1) is locally asymptotically stable, where $E^{*}$ is denoted by (13).

Proof The linearization of system (1) at $E^{*}$ is

$$
\left(\lambda+d_{1}\right)^{2}\left(\lambda+d_{2}\right)^{2} g(\lambda)=0
$$

where

$$
g(\lambda)=\lambda^{2}+\left(\frac{\beta_{1} I_{2}^{*}}{N_{1}^{*}}+d_{1}+\frac{\beta_{2} I_{1}^{*}}{N_{2}^{*}}+d_{2}\right) \lambda+\left(\frac{\beta_{1} I_{2}^{*}}{N_{1}^{*}}+d_{1}\right)\left(\frac{\beta_{2} I_{1}^{*}}{N_{2}^{*}}+d_{2}\right)-d_{1} d_{2} e^{-\lambda\left(\tau_{1}+\tau_{2}\right)} .
$$

It is easy to see that (14) has four negative roots $\lambda_{1}=\lambda_{2}=-d_{1}, \lambda_{3}=\lambda_{4}=-d_{2}$. Therefore, the stability of $E^{*}$ is determined by the distribution of roots of $g(\lambda)=0$.

We define $n_{1}=\frac{\beta_{1} I_{2}^{*}}{N_{1}^{*}}+d_{1}, n_{2}=\frac{\beta_{2} I_{1}^{*}}{N_{2}^{*}}+d_{2}$. Let $\lambda=a+i b$ with $a, b \in \mathbb{R}$ be a root of $g(\lambda)=0$. Then we have

$$
\begin{aligned}
& a^{2}-b^{2}+\left(n_{1}+n_{2}\right) a+n_{1} n_{2}=d_{1} d_{2} e^{-a\left(\tau_{1}+\tau_{2}\right)} \cos \left(\left(\tau_{1}+\tau_{2}\right) b\right), \\
& 2 a b+\left(n_{1}+n_{2}\right) b=-d_{1} d_{2} e^{-a\left(\tau_{1}+\tau_{2}\right)} \sin \left(\left(\tau_{1}+\tau_{2}\right) b\right),
\end{aligned}
$$


it follows that

$$
\begin{aligned}
& \left(a^{2}+b^{2}\right)^{2}+\left(\left(n_{1}+n_{2}\right) a+n_{1} n_{2}\right)^{2}+2 a b^{2}\left(n_{1}+n_{2}\right)+b^{2}\left(n_{1}^{2}+n_{2}^{2}\right) \\
& \quad+2 a^{2}\left(\left(n_{1}+n_{2}\right) a+n_{1} n_{2}\right)-\left(d_{1} d_{2} e^{-a\left(\tau_{1}+\tau_{2}\right)}\right)^{2}=0 .
\end{aligned}
$$

If $a \geq 0$, then the left hand side of (15) is greater than or equal to $\left(n_{1} n_{2}\right)^{2}-\left(d_{1} d_{2} e^{-a\left(\tau_{1}+\tau_{2}\right)}\right)^{2}>$ 0 , a contradiction. This shows that all roots of $g(\lambda)=0$ have negative real parts. Therefore, $E^{*}$ is locally asymptotically stable. This completes the proof of Theorem 5.2.

We can further give the global stability of the endemic equilibrium $E^{*}$.

Theorem 5.3 Assume that $\varepsilon_{2}=\sigma=0$. If $R_{0}>1$, the endemic equilibrium $E^{*}=\left(S_{1}^{*}, E_{1}^{*}, I_{1}^{*}, S_{2}^{*}\right.$, $\left.E_{2}^{*}, I_{2}^{*}\right)$ of system (1) is globally asymptotically stable, where $E^{*}$ is denoted by (13).

Proof In Theorem 5.2, we have given the local stability of $E^{*}$. We now prove the global attractivity of $E^{*}$.

The numbers of the total populations $N_{1}(t)$ and $N_{2}(t)$ satisfy

$$
\begin{aligned}
& \frac{d N_{1}(t)}{d t}=A_{1}-d_{1} N_{1}(t), \\
& \frac{d N_{2}(t)}{d t}=A_{2}-d_{2} N_{2}(t),
\end{aligned}
$$

it follows that

$$
\lim _{t \rightarrow \infty}\left(N_{1}(t), N_{2}(t)\right)=\left(\frac{A_{1}}{d_{1}}, \frac{A_{2}}{d_{2}}\right) .
$$

Therefore, $S_{1}(t), I_{1}(t), S_{2}(t), I_{2}(t)$ satisfy the following limit system:

$$
\begin{aligned}
& \frac{d S_{1}(t)}{d t}=A_{1}-\tilde{\beta}_{1} S_{1}(t) I_{2}(t)-d_{1} S_{1}(t), \\
& \frac{d I_{1}(t)}{d t}=\tilde{\beta}_{1} e^{-d_{1} \tau_{1}} S_{1}\left(t-\tau_{1}\right) I_{2}\left(t-\tau_{1}\right)-d_{1} I_{1}(t), \\
& \frac{d S_{2}(t)}{d t}=A_{2}-\tilde{\beta}_{2} S_{2}(t) I_{1}(t)-d_{2} S_{2}(t), \\
& \frac{d I_{2}(t)}{d t}=\tilde{\beta}_{2} e^{-d_{2} \tau_{2}} S_{2}\left(t-\tau_{2}\right) I_{1}\left(t-\tau_{2}\right)-d_{2} I_{2}(t),
\end{aligned}
$$

where $\tilde{\beta}_{1}=\frac{d_{1} \beta_{1}}{A_{1}}, \tilde{\beta}_{2}=\frac{d_{2} \beta_{2}}{A_{2}}$.

Consider the following Lyapunov functional:

$$
\begin{aligned}
L_{1}(t)= & e^{-d_{1} \tau_{1}}\left[S_{1}-S_{1}^{*}-S_{1}^{*} \ln \left(\frac{S_{1}}{S_{1}^{*}}\right)\right]+I_{1}-I_{1}^{*}-I_{1}^{*} \ln \left(\frac{I_{1}}{I_{1}^{*}}\right) \\
& +\tilde{\beta}_{1} e^{-d_{1} \tau_{1}} \int_{0}^{\tau_{1}}\left[S_{1}(t-r) I_{2}(t-r)-S_{1}^{*} I_{2}^{*}-S_{1}^{*} I_{2}^{*} \ln \left(\frac{S_{1}(t-r) I_{2}(t-r)}{S_{1}^{*} I_{2}^{*}}\right)\right] d r .
\end{aligned}
$$




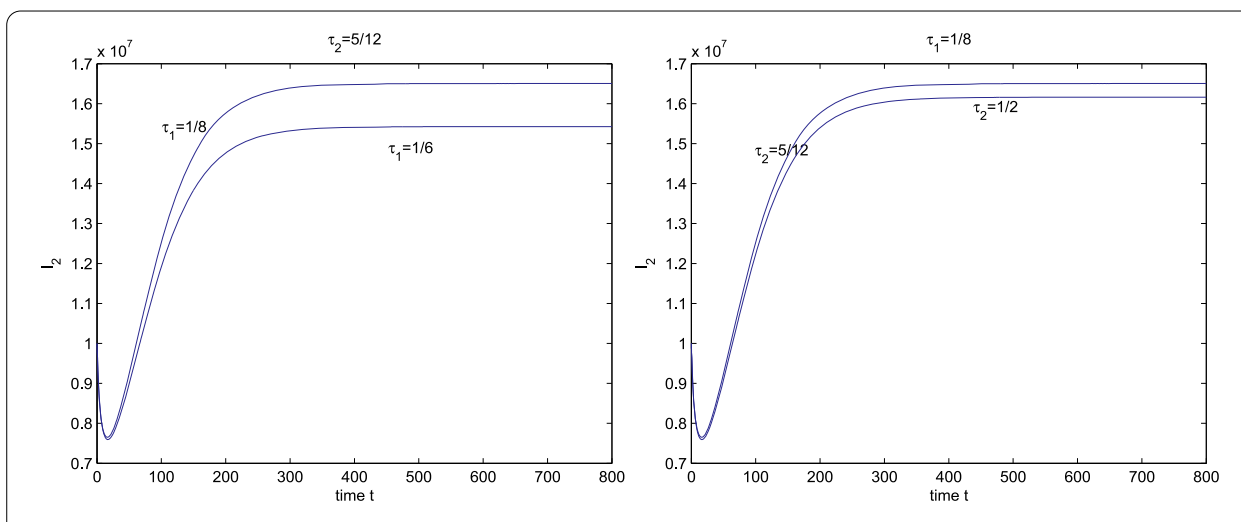

Figure 1 The influence of $\tau_{1}$ and $\tau_{2}$ on the number of infected intermediate hosts $I_{2}$.

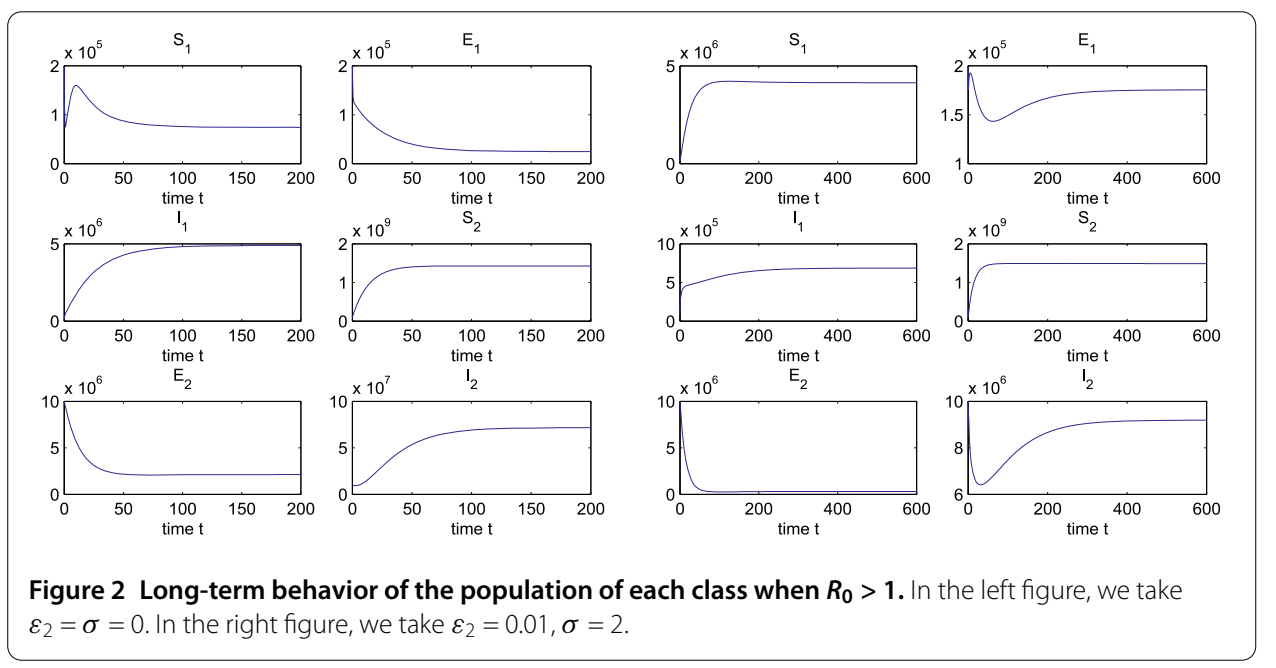

Note that $1-x \leq \ln \left(\frac{1}{x}\right), x \in R^{+}$, with equality only if $x=1$, then the derivative of $L_{1}$ along the solution of (16) satisfies

$$
\begin{aligned}
\frac{d L_{1}}{d t}= & -d_{1} e^{-d_{1} \tau_{1}} \frac{\left(S_{1}-S_{1}^{*}\right)^{2}}{S_{1}}+\tilde{\beta}_{1} e^{-d_{1} \tau_{1}} S_{1}^{*} I_{2}^{*}\left(1-\frac{S_{1}^{*}}{S_{1}}+\frac{I_{2}}{I_{2}^{*}}-\frac{I_{1}}{I_{1}^{*}}\right) \\
& +\tilde{\beta}_{1} e^{-d_{1} \tau_{1}} S_{1}^{*} I_{2}^{*}\left(1-\frac{S_{1}\left(t-\tau_{1}\right) I_{2}\left(t-\tau_{1}\right) I_{1}^{*}}{S_{1}^{*} I_{2}^{*} I_{1}}\right. \\
& \left.+\ln \left(S_{1}\left(t-\tau_{1}\right) I_{2}\left(t-\tau_{1}\right)\right)-\ln \left(S_{1}(t) I_{2}(t)\right)\right) \\
\leq & \tilde{\beta}_{1} e^{-d_{1} \tau_{1}} S_{1}^{*} I_{2}^{*}\left(\ln \left(\frac{S_{1}}{S_{1}^{*}}\right)+\frac{I_{2}}{I_{2}^{*}}-\frac{I_{1}}{I_{1}^{*}}+\ln \left(\frac{S_{1}^{*} I_{2}^{*} I_{1}}{S_{1}\left(t-\tau_{1}\right) I_{2}\left(t-\tau_{1}\right) I_{1}^{*}}\right)\right. \\
& \left.+\ln \left(S_{1}\left(t-\tau_{1}\right) I_{2}\left(t-\tau_{1}\right)\right)-\ln \left(S_{1}(t) I_{2}(t)\right)\right) \\
= & \tilde{\beta}_{1} e^{-d_{1} \tau_{1}} S_{1}^{*} I_{2}^{*}\left(\ln \left(\frac{I_{2}^{*} I_{1}}{I_{1}^{*} I_{2}}\right)+\frac{I_{2}}{I_{2}^{*}}-\frac{I_{1}}{I_{1}^{*}}\right) .
\end{aligned}
$$



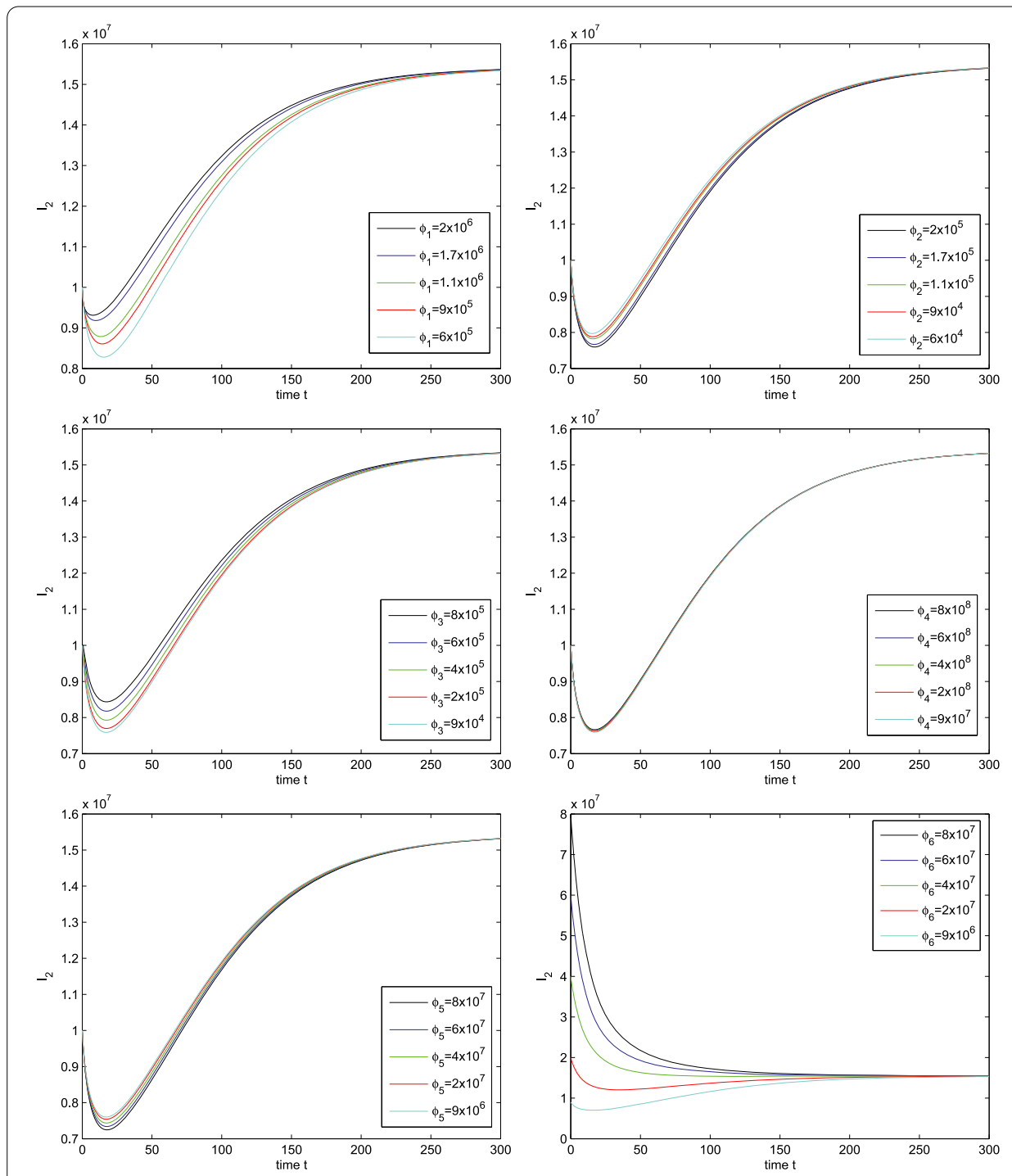

Figure 3 The influence of the initial conditions on the number of infected intermediate hosts, $I_{2}$.

If we construct another Lyapunov functional,

$$
\begin{aligned}
L_{2}(t)= & e^{-d_{2} \tau_{2}}\left[S_{2}-S_{2}^{*}-S_{2}^{*} \ln \left(\frac{S_{2}}{S_{2}^{*}}\right)\right]+I_{2}-I_{2}^{*}-I_{2}^{*} \ln \left(\frac{I_{2}}{I_{2}^{*}}\right) \\
& +\tilde{\beta}_{2} e^{-d_{2} \tau_{2}} \int_{0}^{\tau_{2}}\left[S_{2}(t-r) I_{1}(t-r)-S_{2}^{*} I_{1}^{*}-S_{2}^{*} I_{1}^{*} \ln \left(\frac{S_{2}(t-r) I_{1}(t-r)}{S_{2}^{*} I_{1}^{*}}\right)\right] d r,
\end{aligned}
$$

by a similar computation to $\frac{d L_{1}}{d t}$, we obtain

$$
\frac{d L_{2}}{d t} \leq \tilde{\beta}_{2} e^{-d_{2} \tau_{2}} S_{2}^{*} I_{1}^{*}\left(\ln \left(\frac{I_{1}^{*} I_{2}}{I_{2}^{*} I_{1}}\right)+\frac{I_{1}}{I_{1}^{*}}-\frac{I_{2}}{I_{2}^{*}}\right)
$$

Finally we choose the Lyapunov functional

$$
L(t)=\tilde{\beta}_{2} e^{-d_{2} \tau_{2}} S_{2}^{*} I_{1}^{*} L_{1}(t)+\tilde{\beta}_{1} e^{-d_{1} \tau_{1}} S_{1}^{*} I_{2}^{*} L_{2}(t),
$$



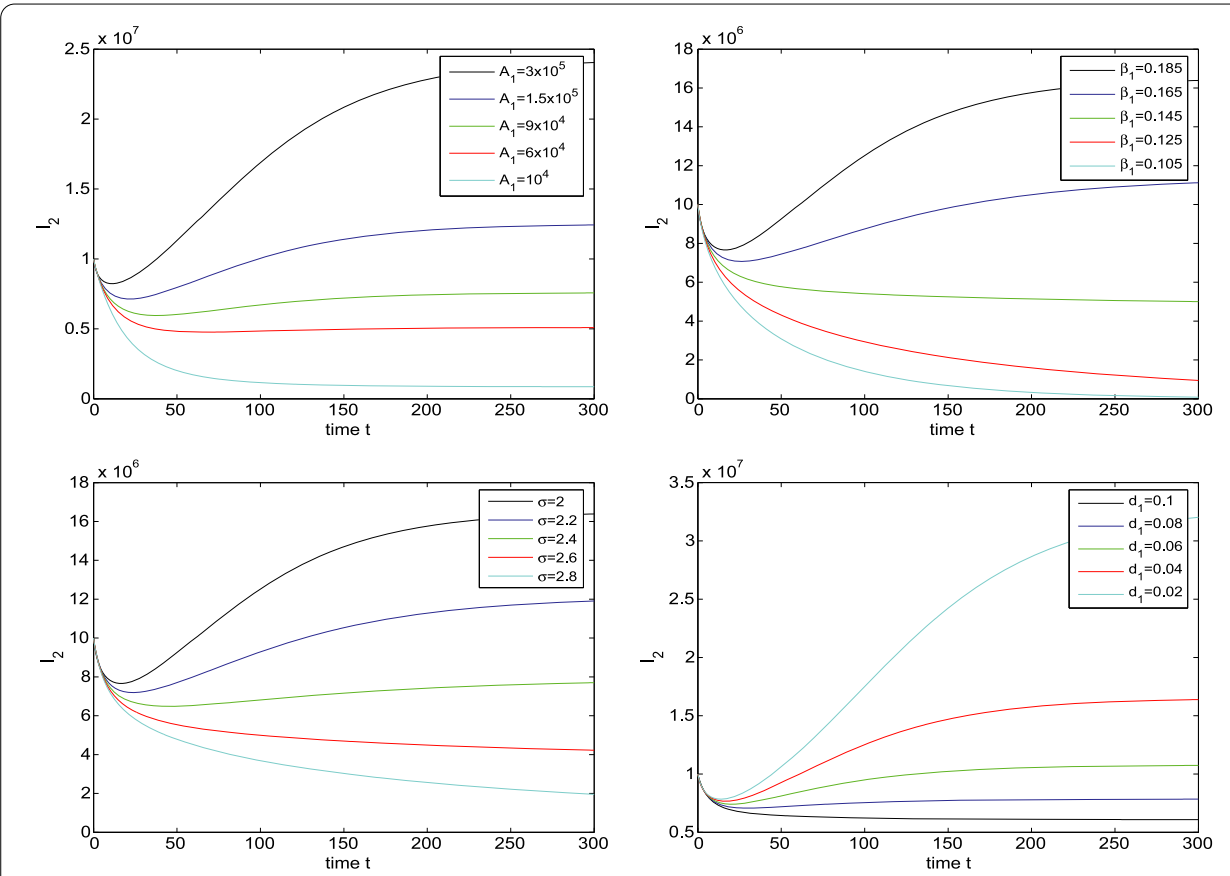

Figure 4 The influence of parameters $A_{1}, \beta_{1}, \sigma$, and $d_{1}$ on the number of infected intermediate hosts, $I_{2}$.

then we get

$$
\frac{d L}{d t} \leq 0
$$

Let $M \subset E=\left\{\left(S_{1}, I_{1}, S_{2}, I_{2}\right): \frac{d L}{d t}=0\right\}$ be the largest invariant set with respect to system (16), we can show that $M=\left\{\left(S_{1}^{*}, I_{1}^{*}, S_{2}^{*}, I_{2}^{*}\right)\right\}$. By the Lasalle invariance principle, $\left(S_{1}^{*}, I_{1}^{*}, S_{2}^{*}, I_{2}^{*}\right)$ is globally attractive. From (2) and (3), we have $E_{1}(t) \rightarrow E_{1}^{*}, E_{2}(t) \rightarrow E_{2}^{*}$ as $t \rightarrow \infty$. Now we see that $E^{*}=\left(S_{1}^{*}, E_{1}^{*}, I_{1}^{*}, S_{2}^{*}, E_{2}^{*}, I_{2}^{*}\right)$ is globally attractive. This completes the proof.

\section{Numerical simulations}

In this section, we carry out numerical simulations to illustrate our analytic results. Since all the parameters are not easy to find, we will assume some parameters.

In view of [22], we fix $\beta_{1}=0.185$ year $^{-1}, d_{1}=0.04$ year $^{-1}, d_{2}=0.07$ year $^{-1}$, we first choose $\sigma=2 \operatorname{year}^{-1}, \varepsilon_{2}=0 \operatorname{year}^{-1}$ (see [14]), $\tau_{1}=\frac{1}{8} \sim \frac{1}{6}$ year, $\tau_{2}=\frac{5}{12} \sim \frac{1}{2}$ year (see [16]).

First we take $A_{1}=2 \times 10^{5}$ year $^{-1}, A_{2}=1.05 \times 10^{8}$ year $^{-1}, \beta_{2}=1.11$ year $^{-1}$. Fixing $\tau_{2}=$ $5 / 12$ year, we vary $\tau_{1}$ from $\frac{1}{8}$ year to $\frac{1}{6}$ year, and from Figure 1 we see the disease of the intermediate hosts population $I_{2}$ persists; as $\tau_{1}$ increases, the infection level becomes lower. If we fix $\tau_{1}=1 / 8$ year and vary $\tau_{2}$ from $\frac{5}{12}$ year to $\frac{1}{2}$ year, also Figure 1 shows that as $\tau_{2}$ increases, $I_{2}$ decreases. This figure implies that large time delays are beneficial to disease control. Also we see that $\tau_{1}$ has a bigger impact than $\tau_{2}$ on the infection level of $I_{2}$.

Then we take $\varepsilon_{2}=\sigma=0$; we get $R_{0}=8.4188$, and by Theorem 5.3 , we find that $E^{*}$ is globally asymptotically stable (see the left figure of Figure 2). But if we choose $\varepsilon_{2}=0.01$, $\sigma=2$, then we get $R_{0}=1.1027$, and from the right figure of Figure 2, we see that there is still an endemic equilibrium $E^{*}$ which is globally asymptotically stable. 

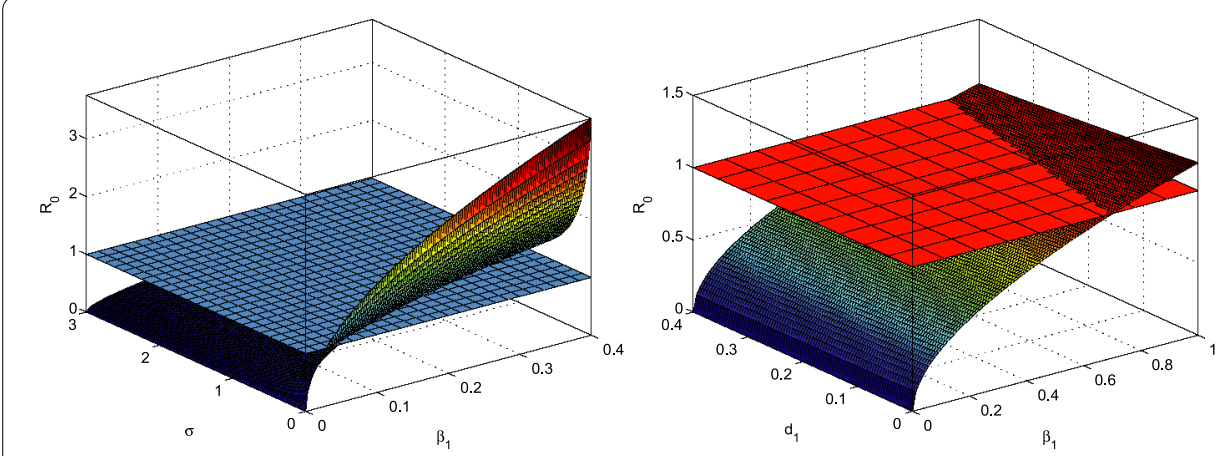

Figure 5 Surface plots of $R_{0}$ as a function of $\beta_{1}, d_{1}$, and $\sigma$.

We now investigate the impact of the initial conditions on the number of infected intermediate hosts, $I_{2}(t)$. From Figure 3 , we can see that $\phi_{1}, \phi_{3}, \phi_{6}$ have a stronger impact than the other initial values, $\phi_{4}$ has almost no influence on $I_{2}(t)$. These figures show that it is very important to control the amount of susceptible and infected definitive hosts.

Next we want to see the influence of different parameters on the amount of infected intermediate hosts $I_{2}(t)$. The influence of $A_{1}, \beta_{1}, \sigma, d_{1}$ on $I_{2}(t)$ is shown in Figure 4. From the expression of $R_{0}$, we can see that $R_{0}$ decreases if $\beta_{1}$ decreases, or $\sigma$ increases, or $d_{1}$ increases. Figure 4 also verifies this.

Figure 5 depicts the relationship of $R_{0}$ as a function of $\beta_{1}, d_{1}$, and $\sigma$, we can see that $R_{0}$ is not always less than 1 .

Remark 6.1 Although $A_{1}$ does not affect the number of $R_{0}$, reducing $A_{1}$ can decrease the infection level of $I_{2}$ (see Figure 4).

Based on the above analysis, we now give some control strategies by adjusting the parameters $A_{1}, \beta_{1}, \sigma$, and $d_{1}$. (1) Decrease $A_{1}$ by reducing the birth rate of newborn puppies. (2) $\beta_{1}$ can be reduced by the following measures. Livestock slaughtering regulations and health education should be implemented in endemic areas. Infected offal should be treated harmlessly. Definitive hosts should be barred from slaughter houses. (3) $\sigma$ can be increased through increasing the frequency of anticestodal drugs (e.g., praziquantel). (4) In order to increase $d_{1}$, we can kill the infected definitive hosts and the stray dog populations.

Competing interests

The authors declare that they have no competing interests.

\section{Authors' contributions}

$J \mathrm{~L}$ proposed the model and completed the main part of this manuscript, $\mathrm{LL}$ checked all the theorems and polished the language, XF enhanced the revised version, and JF revised the numerical simulation part. All the authors read and approved the manuscript.

\section{Author details}

'School of Science, Xi'an Polytechnic University, Xi'an, 710048, P.R. China. ${ }^{2}$ School of Mathematics and Statistics, Henan University of Science and Technology, Luoyang, 471023, P.R. China. ${ }^{3}$ Department of Applied Mathematics, Yuncheng University, Yuncheng, 044000, P.R. China.

\section{Acknowledgements}

The authors are grateful to both reviewers for their helpful suggestions and comments. This work was supported in part by the National Nature Science Foundation of China (NSFC 11101323, 11101127 and 11302158), the Natural Science Basic Research Plan in Shaanxi Province of China (2014JQ1038), Scientific Research Program Funded by Shaanxi Provincial Education Department (11JK0469), the Research Project at Yuncheng University (SWSX201404). 
Received: 13 October 2014 Accepted: 2 January 2015 Published online: 28 March 2015

\section{References}

1. Echinococcosis Fact sheet $N^{\circ} 377$, World Health Organization, March 2014. Retrieved 19 March 2014

2. Lozano, R, Naghavi, M, Foreman, K: Global and regional mortality from 235 causes of death for 20 age groups in 1990 and 2010: a systematic analysis for the Global Burden of Disease Study 2010. Lancet 380, 2095-2128 (2012)

3. Zhu, W: Progress and present status of hydatid disease control in China in the recent eight decades. In: Hami Prefectural Institute of Scientific and Technological Information, Hami, China, p. 5 (1985) (in Chinese)

4. Chu, K: Multiple pulmonary hydatid disease in left lung: report of a case. In: A Collection of Abstracts of Papers Presented at the Second National Symposium on Hydatidosis, Urumqi, China, p. 73 (1992) (in Chinese)

5. Chai, J: Epidemiological studies on cystic echinococcosis in China-a review. Biomed. Environ. Sci. 8, $122-136$ (1995)

6. Qiu, J, Liu, F, Schantz, P, Ito, A, et al.: Epidemiological study on human hydatidosis in Tibetan region of western Sichuan. Chin. J. Zoonoses 16, 77-80 (2000) (in Chinese)

7. Li, T, Qiu, J, Yang, W, Craig, P, et al.: Echinococcosis in Tibetan populations, western Sichuan province, China. Emerg. Infect. Dis. 11, 1866-1873 (2005)

8. Wang, Z, Wang, $X$, Liu, X: Echinococcosis in China, a review of the epidemiology of Echinococcus spp. EcoHealth 5, $115-126(2008)$

9. Azlaf, R, Dakkak, A, Chentou, A, Berrahmani, M: Modelling the transmission of Echinococcus granulosus in dogs in the northwest and in the southwest of Morocco. Vet. Parasitol. 145, 297-303 (2007)

10. Torgerson, P, Burtisurnov, K, Shaikenov, B, et al.: Modelling the transmission dynamics of Echinococcus granulosus in sheep and cattle in Kazakhstan. Vet. Parasitol. 114, 143-153 (2003)

11. Torgerson, P, Ziadinov, I, Aknazarov, D, Nurgaziev, R, Deplazes, P: Modelling the age variation of larval protoscoleces of Echinococcus granulosus in sheep. Int. J. Parasitol. 39(9), 1031-1035 (2009)

12. Huang, L, Huang, Y, Wang, Q, Xiao, N, Yi, D, Yu, W, Qiu, D: An agent-based model for control strategies of Echinococcus granulosus. Vet. Parasitol. 179(1-3), 84-91 (2011)

13. Kato, N, Kotani, K, Ueno, S, Matsuda, H: Optimal risk management of human alveolar echinococcosis with vermifuge. J. Theor. Biol. 267, 265-271 (2010)

14. Wang, K, Zhang, X, Jin, Z, Ma, H, Teng, Z, Wang, L: Modeling and analysis of the transmission of echinococcosis with application to Xinjiang Uygur Autonomous Region of China. J. Theor. Biol. 333, 78-90 (2013)

15. Du, S, Wang, L, Zhang, X, Wang, K: A echinococcosis model with saturation incidence. Math. Pract. Theory 43 269-273 (2013)

16. Wang, C, Li, J, LV, G, He, F, Zhang, S: The epidemic process, symptoms and harm of hydatid disease. Vet. Orientation 6, 24-25 (2011)

17. Wang, Z, Zhao, X-Q: Global dynamics of a time-delayed dengue transmission model. Can. Appl. Math. Q. 20, 89-113 (2012)

18. Thieme, HR: Convergence results and a Poincare-Bendixson trichotomy for asymptotically autonomous differential equations. J. Math. Biol. 30, 755-763 (1992)

19. Hale, JK: Asymptotic Behavior of Dissipative Systems. Am. Math. Soc., Providence (1988)

20. Smith, HL: Monotone Dynamical Systems: An Introduction to the Theory of Competitive and Cooperative Systems. Math. Surveys Monogr., vol. 41. Am. Math. Soc., Providence (1995)

21. Smith, HL, Zhao, X-Q: Robust persistence for semidynamical systems. Nonlinear Anal., Theory Methods Appl. 47 6169-6179 (2001)

22. Zhao, Y, Kang, Y: Mathematical modelling and controlling of echinococcosis spread. Chin. J. Vet. Parasitol. 19, 81-86 (2011)

\section{Submit your manuscript to a SpringerOpen ${ }^{\odot}$ journal and benefit from:}

- Convenient online submission

- Rigorous peer review

- Immediate publication on acceptance

Open access: articles freely available online

- High visibility within the field

- Retaining the copyright to your article 